\title{
ANEURISMA DE ARTÉRIA HEPÁTICA SIMULANDO LESÃO EM CABEÇA DE PÂNCREAS: RELATO DE CASO*
}

\author{
Oswaldo Luiz Pinto ${ }^{1}$, Olger de Souza Tornin ${ }^{2}$, Renato Assayag Botelho ${ }^{2}$, Marcelo Carneiro \\ Menezes ${ }^{2}$, José Francisco Sales Chagas ${ }^{3}$, José Gonzaga Camargo ${ }^{1}$, Ricardo Pires de Souza ${ }^{4}$
}

\begin{abstract}
Resumo 0 objetivo deste estudo é relatar um caso raro de aneurisma da artéria hepática simulando lesão em cabeça de pâncreas. É importante a análise precisa por tomografia computadorizada e ressonância magnética, pois desempenham papel importante no planejamento terapêutico e influenciam também o prognóstico.

Unitermos: Artéria hepática; Aneurisma; Pâncreas; Tomografia computadorizada; Ressonância magnética.
\end{abstract}

Abstract Hepatic artery aneurysm simulating a lesion in the head of the pancreas: a case report.

The aim of this study is to report a rare case of hepatic artery aneurysm simulating a lesion in the head of the pancreas. A precise evaluation using computed tomography and magnetic resonance imaging is important in the treatment planning, which will influence the prognosis.

Key words: Hepatic artery; Aneurysm; Pancreas; Computed tomography; Magnetic resonance imaging.

\section{INTRODUÇÃO}

Os aneurismas da artéria hepática são raros e representam $20 \%$ dos aneurismas viscerais. Estas lesões localizam-se freqüentemente na porção extra-hepática e têm dimensões variáveis, raramente menores que $2 \mathrm{~cm}$ ou maiores que $10 \mathrm{~cm}$. Habitualmente, os aneurismas da artéria hepática são detectados na vida adulta, sendo mais prevalentes no sexo masculino (4:1). O sintoma inicial mais comum é a dor no andar superior do abdome, presente em $75 \%$ dos casos, podendo preceder em meses a ruptura ${ }^{(1-3)}$.

Embora a grande maioria tenha evolução assintomática, consistindo, muitas vezes, em achados diagnósticos incidentais, sua ruptura apresenta elevada mortalidade, com apresentações clínicas dramáticas ${ }^{(4)}$. Suas causas incluem arteriosclerose, trau-

\footnotetext{
* Trabalho realizado no Serviço de Diagnóstico por Imagem do Complexo Hospitalar Heliópolis, São Paulo, SP.

1. Médicos, Professores do Departamento de Cirurgia da Pontifícia Universidade Católica de Campinas.

2. Médicos Residentes em Radiologia e Diagnóstico por Imagem do Complexo Hospitalar Heliópolis.

3. Professor do Curso de Pós-Graduação em Ciências da Saúde do Complexo Hospitalar Heliópolis, Doutor em Medicina pela Escola Paulista de Medicina/Universidade Federal de São Paulo (EPM/Unifesp).

4. Professor do Curso de Pós-Graduação em Ciências da Saúde e Coordenador da Residência Médica em Radiologia e Diagnóstico por Imagem do Complexo Hospitalar Heliópolis, Doutor em Radiologia pela Universidade de São Paulo (USP).

Endereço para correspondência: Dr. Olger de Souza Tornin. Rua Cônego Xavier, 276, $1^{\circ}$ andar, Secretaria do RX, Bairro Sacomã. São Paulo, SP, 04231-030. E-mail: olger@ibest.com.br

Recebido para publicação em 20/12/2004. Aceito, após revisão, em 7/1/2005.
}

ma, cirurgia, inflamação, infecção, necrose da camada média, doenças do colágeno, arterites e anomalias congênitas ${ }^{(4)}$.

O tratamento do aneurisma da artéria hepática tem indicação precisa, devido à frequiência com que pode ocorrer ruptura acompanhada de elevada mortalidade ${ }^{(1-3)}$.

Pela sua baixa incidência, quadro clínico inespecífico, e pela raridade descrita na literatura mimetizando lesão neoplásica em cabeça de pâncreas, os autores julgam oportuno o relato do caso, tratado com sucesso por cirurgia.

\section{RELATO DO CASO}

Paciente de 56 anos de idade, sexo masculino, caucasóide, natural de Franca, SP. Procurou atendimento médico em agosto de 2003 com queixa de desconforto abdominal, principalmente na região epigástrica e periumbilical, acompanhado de plenitude e meteorismo.

Ao exame físico, apresentava estado geral e nutricional normais, hemodinamicamente estável, mucosas coradas, hidratadas, anictéricas, ausência de linfonodomegalias. O abdome era plano e discretamente doloroso à palpação profunda do epigástrio e mesogástrio.

Como antecedentes, apresentava esofagite e gastrite leve de corpo e antro, diagnosticados à esofagogastroduodenoscopia prévia. Referia também ser portador de doença diverticular dos cólons, forma hi- pertônica, em exame colonoscópico realizado anteriormente.

Para avaliação do fígado, vesícula biliar, vias biliares intra e extra-hepática e pâncreas, foi solicitada ultra-sonografia abdominal, que revelou presença de processo hipoecóico, de contornos regulares, medindo $3,8 \times 2,7 \mathrm{~cm}$, em projeção da cabeça do pâncreas (Figura 1).

Com o objetivo de melhor definição da lesão e seu estadiamento, realizou-se tomografia computadorizada de abdome, sem (Figura 2) e com (Figura 3) contraste, esta última mostrando lesão complexa, consistindo de lesão hiperdensa em relação ao parênquima pancreático, arredondada, bem delimitada, medindo $4,3 \times 3,8$ $\times 2,8 \mathrm{~cm}$.

Foram realizados exames laboratoriais - ALT, AST, fosfatase alcalina, gama GT, bilirrubinas, CA 19-9 -, todos com resultados normais.

Observando que os achados de imagem não correspondiam com a clínica e exame físico do paciente, e a densidade da lesão à tomografia sugeria processo vascular, foi solicitada ressonância magnética (Figura 4) e, também, angiorressonância magnética (Figura 5), que evidenciaram dilatação aneurismática sacular na artéria hepática, medindo $33,8 \times 32,1 \mathrm{~mm}$, comprimindo posteriormente a veia porta adjacente.

Como planejamento terapêutico prévio, realizou-se angiografia seletiva no tronco celíaco (Figura 6). 


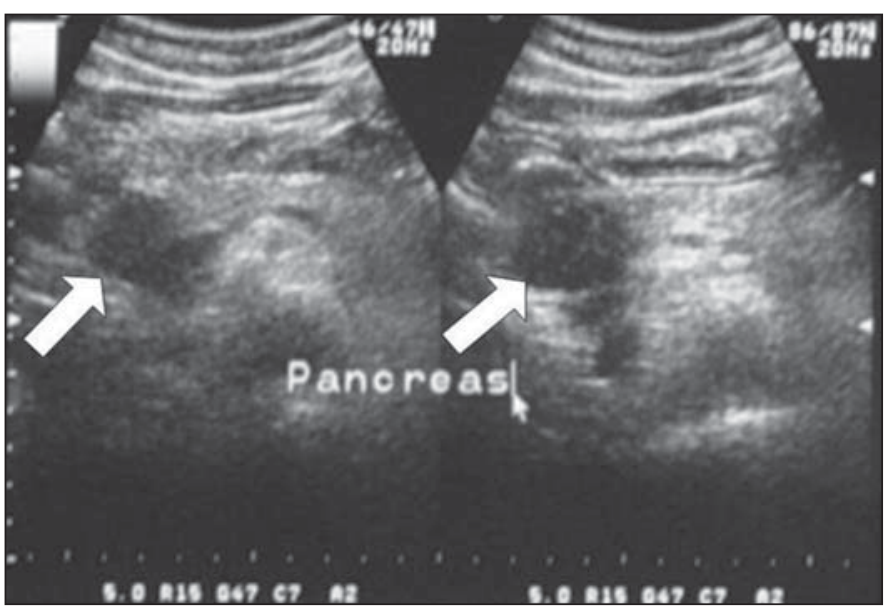

Figura 1. Ultra-sonografia abdominal evidencia imagem hipoecóica, de limites bem definidos e contornos regulares, em topografia da cabeça do pâncreas.

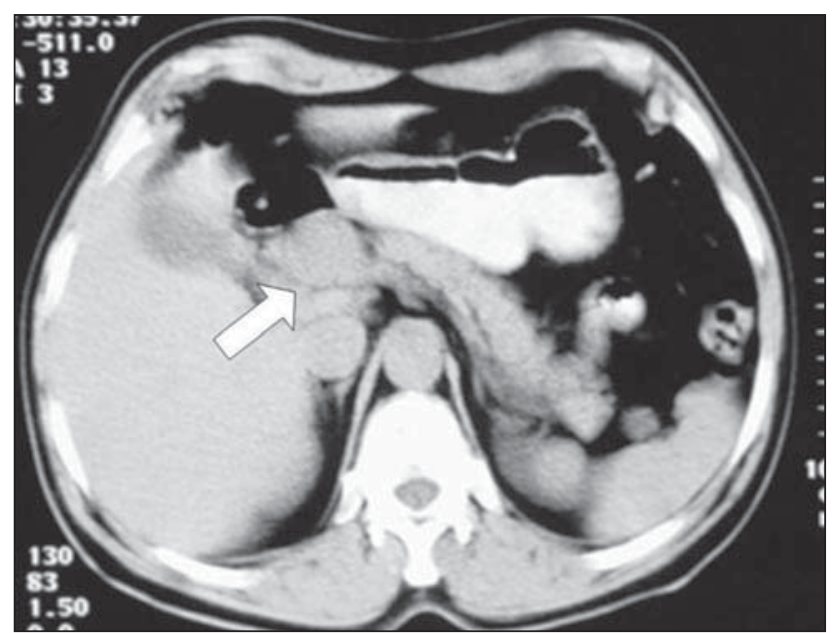

Figura 2. Tomografia computadorizada (fase não-contrastada). Obsenvar o aumento volumétrico em topografia da cabeça pancreática.

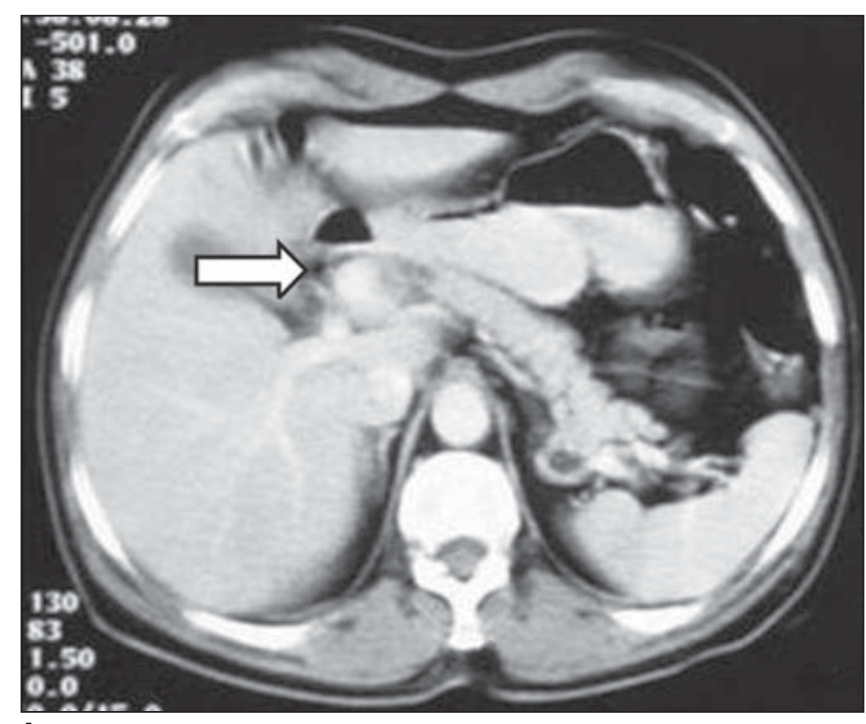

A

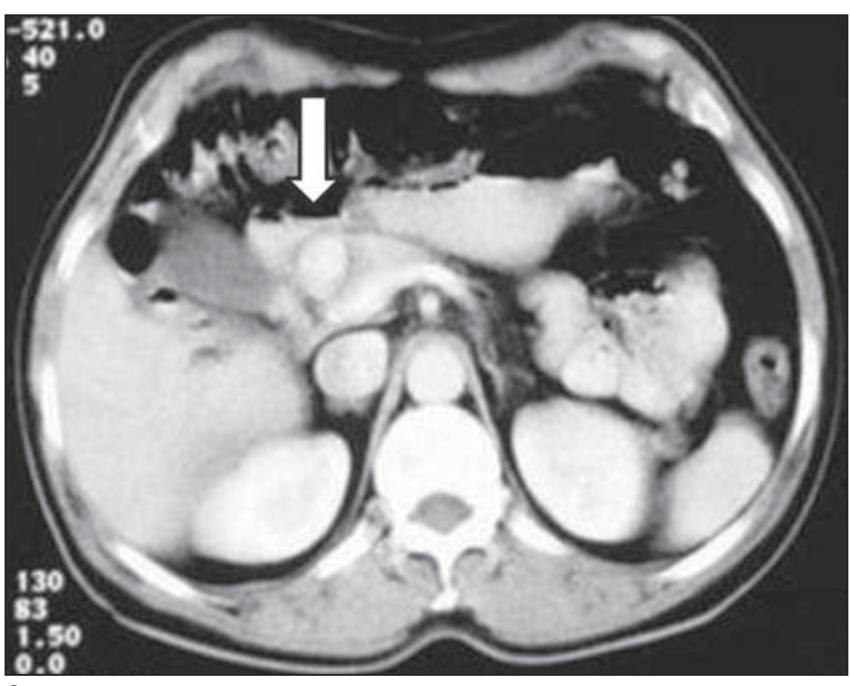

C

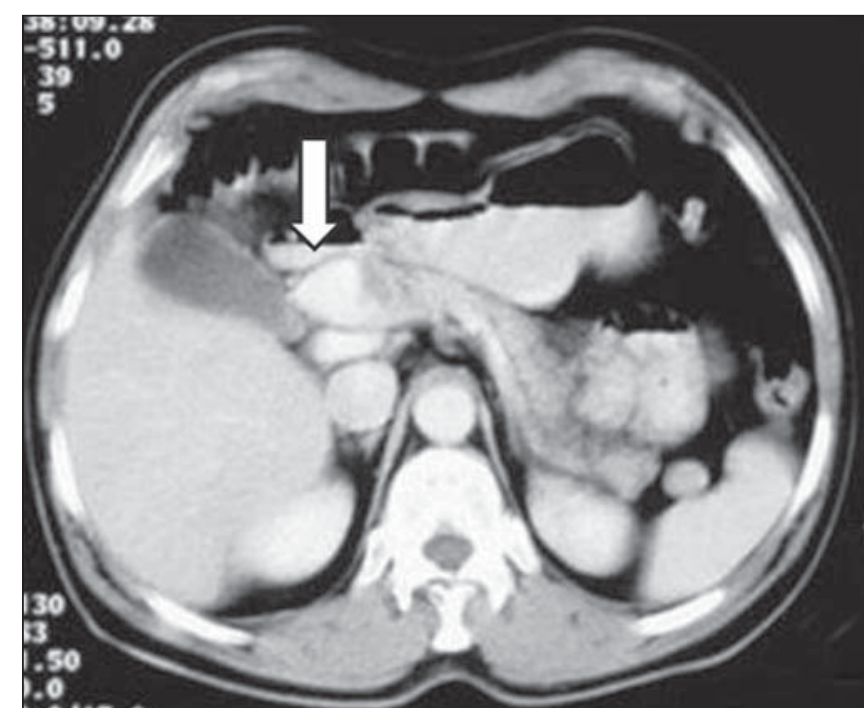

B

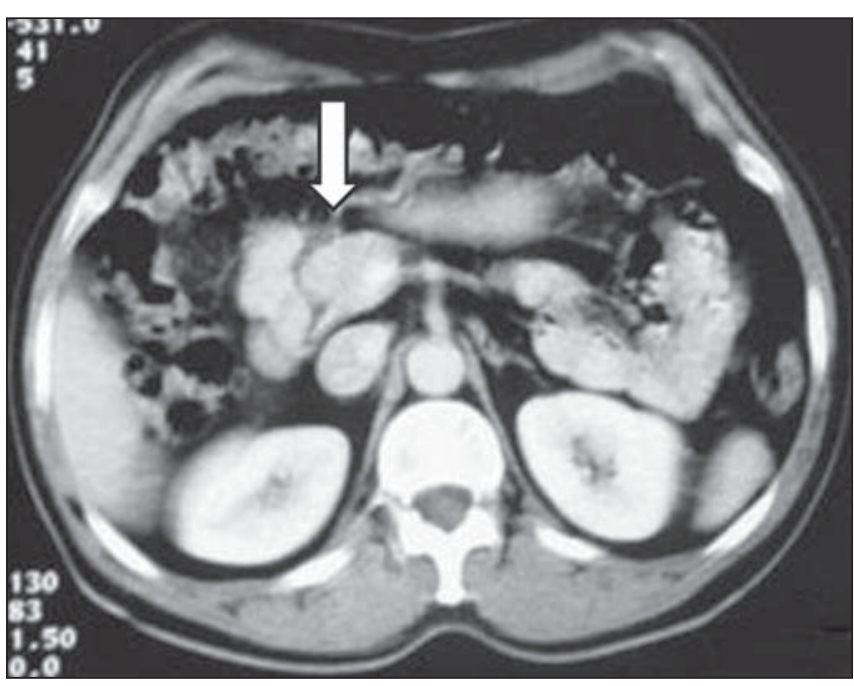

D

Figura 3. Tomografia computadorizada (fase contrastada). 0 importante realce pelo meio de contraste sugere lesão de natureza vascular. 
Figura 4. Ressonância magnética. Imagem arredondada com sinal heterogêneo, em FAST T2, na porção medial da cabeça pancreática.

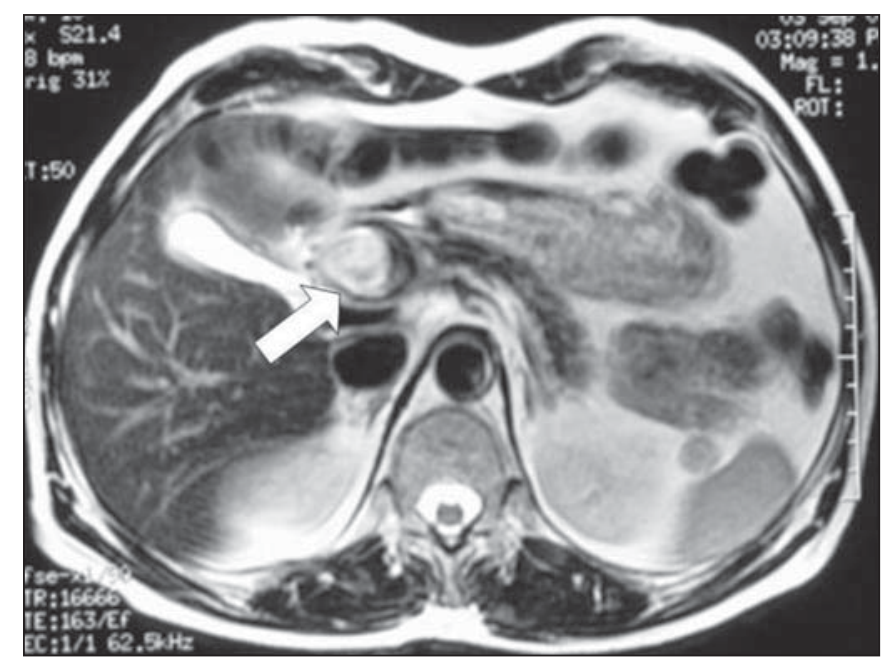

Em novembro de 2003 o paciente foi submetido a procedimento cirúrgico, no qual foram realizadas colecistectomia e ressecção do aneurisma com interposição de safena autóloga, tendo o paciente recebido alta hospitalar no quinto dia de pósoperatório. No pós-operatório tardio de um ano, encontrava-se assintomático.

\section{DISCUSSÃO}

Os aneurismas da artéria hepática são raros e representam $16 \%$ a $20 \%$ dos aneurismas viscerais. Simulando lesões expansivas na cabeça pancreática, são poucos os relatos na literatura ${ }^{(\mathbf{5})}$

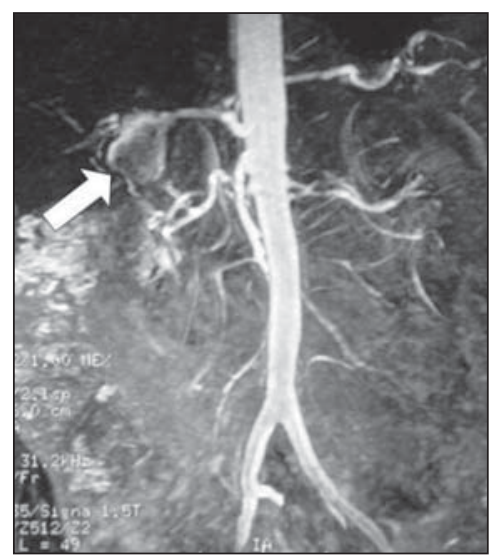

A

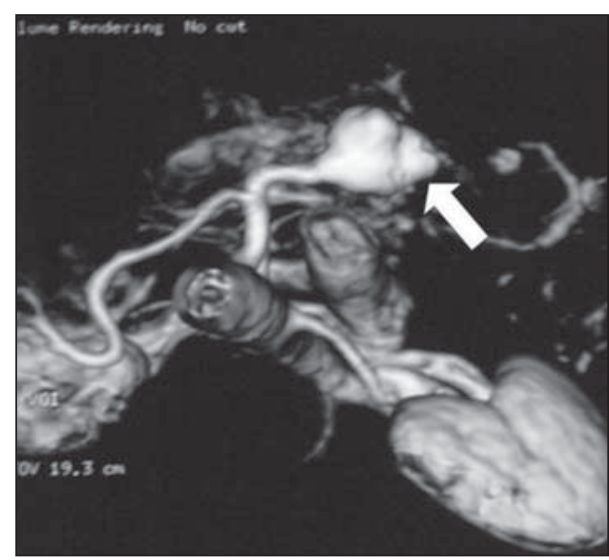

B

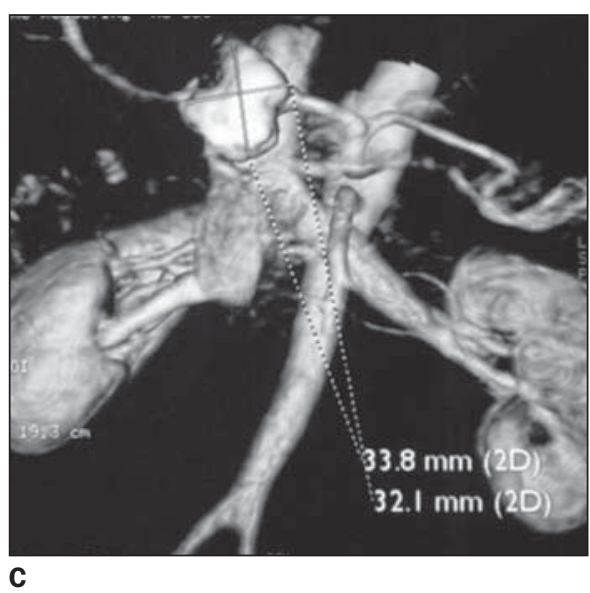

C

Figura 5. Angiografia por ressonância magnética. Dilatação aneurismática da artéria hepática com contornos lobulados, medindo 3,3 × 3,2 cm.

Figura 6. Angiografia seletiva do tronco celíaco. Dilatação aneurismática da artéria hepática.

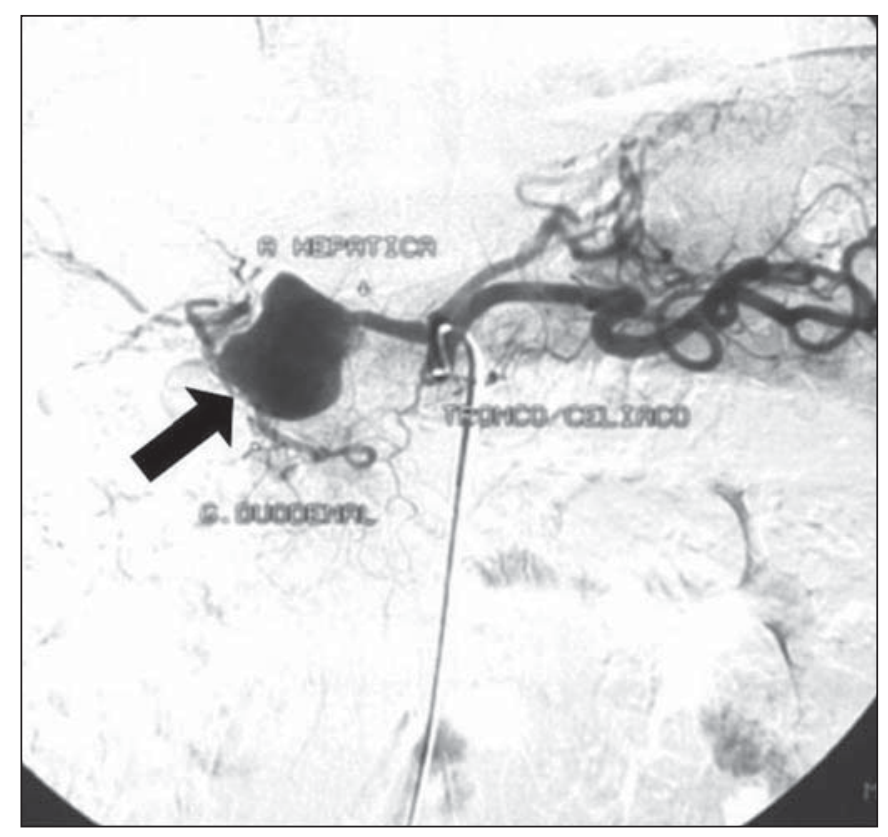

A crescente utilização dos métodos de imagem, especificamente a ultra-sonografia, pela facilidade de execução, não ser invasiva e relativo baixo custo, tem permitido o diagnóstico sugestivo de casos assintomáticos. O diagnóstico pode ser confirmado pela tomografia computadorizada após injeção de contraste, arteriografia hepática ou por ressonância magnética ${ }^{(2,3)}$.

Para as lesões intra-hepáticas, as opções de tratamento incluem embolização angiográfica com "gelfoam" ou "cianoacrilato", a excisão da lesão pela ressecção do segmento hepático envolvido e a ligadura do vaso nutridor do aneurisma ${ }^{(1,3)}$.

Os aneurismas da artéria hepática comum, quando proximais à artéria gastroduodenal, podem ser ligados com segurança. Já os aneurismas distais à artéria gastroduodenal devem ser tratados com 
ressecção cirúrgica e reconstrução, visto que a ligadura neste nível compromete a circulação colateral, podendo resultar em isquemia hepática ${ }^{(3)}$.

O presente caso mostra a importância da história clínica e exame físico, corroborados pelos exames laboratoriais, que não sugeriam processo expansivo em cabeça de pâncreas, levando os autores a buscar o diagnóstico definitivo, que foi possível com a tomografia computadorizada, angiorressonância magnética e aortografia abdominal.
Salientamos que a utilização da ultrasonografia com Doppler e contraste endovenoso nos exames tomográficos com suspeita de lesões vasculares favorece o diagnóstico preciso.

\section{REFERÊNCIAS}

1. Schafer DF, Sorrel MF. Vascular diseases of the liver. In: Feldman M, Scharshimidt BF, Slazenger MH, editors. Sleisenger \& Fordtran Gastrointestinal and liver disease. Pathophysiology, diagnosis, management. 6th ed. Philadelphia: WB Saunders, $1988 ; 1188-1198$.

2. Téres J. Hepatic arteries. In: Bircher J, Benhamon JP, McIntire N, Rizzeto M, Rodés J, editors. Oxford
Textbook of clinical hepatology. 2nd ed. Oxford: Oxford University Press, 1999;1459-1461.

3. Parolim MB, Lopes RW. Doenças vasculares do fígado. In: Kalil AN, Coelho JUC, Strauss E. Fígado e vias biliares: clínica e cirurgia. Rio de Janeiro: Revinter, 2001.

4. Garcia RG, Kamya CA, Ishikawa WY, Menezes MR, Cerri GG. Aneurismas arteriais esplâncnicos - experiência do Serviço de Radiologia de Emergência - Departamento de Radiologia da Faculdade de Medicina da USP. Disponível em: http://www. hcnet.usp.br/inrad/departamento/radiops.htm

5. Nemeth GG, Elijovich F, Schanzer H. Hepatic artery aneurysm simulating a pancreatic mass. J Cardiovasc Surg (Torino) 1992;33:629-631. 